PROCEEDINGS OF THE

AMERICAN MATHEMATICAL SOCIETY

Volume 126, Number 12, December 1998, Pages 3597-3605

S 0002-9939(98)04934-X

\title{
UNITARILY-INVARIANT LINEAR SPACES IN C*-ALGEBRAS
}

\author{
L. W. MARCOUX AND G. J. MURPHY
}

(Communicated by Palle E. T. Jorgensen)

\begin{abstract}
Characterisations and containment results are given for linear subspaces of a unital $\mathrm{C}^{*}$-algebra that are invariant under conjugation by sets of unitary elements of the algebra. The (unitarily-invariant) linear span of the projections in a simple, unital $\mathrm{C}^{*}$-algebra having non-scalar projections is shown to contain all additive commutators of the algebra and, in certain cases, to be equal to the algebra.
\end{abstract}

\section{INTRODUCTION}

It is a well known result in $\mathrm{C}^{*}$-algebra theory that a closed linear subspace of a unital $\mathrm{C}^{*}$-algebra that is invariant under conjugation by all unitary elements is necessarily a Lie ideal of the algebra. By using a theorem of I.N. Herstein, one can deduce containment results for the invariant space-see Theorem 2.1 below. In this paper we consider such invariant linear spaces and also linear spaces that are not necessarily closed on which a weaker requirement is imposed: they are merely assumed to be invariant under conjugation by finite-order unitaries. We characterise such spaces and show that, in certain cases, they also satisfy similar containment conditions: they are frequently either contained in the centre of the algebra or contain all the (additive) commutators of the algebra.

A unitarily-invariant linear space of particular importance for this paper is the linear span of the projections. The two questions concerning this space that we address are: (1) When does this space contain all commutators (this indicates that the space is "large"); and (2) When is this space actually equal to the algebra? Of course, it is well known that for many large classes of $\mathrm{C}^{*}$-algebras, such as von Neumann algebras, AF-algebras and, more generally, $\mathrm{C}^{*}$-algebras of real rank zero, the projections have dense linear span. In some cases, as we shall see below, the projections linearly span the algebra-one does not have to take a closure. However, it usually requires significantly more work to show the linear span of the projections is equal to the algebra rather than is merely dense in the algebra. Our analysis of this question leads to the strengthening of some earlier results of G.K. Pedersen, who, in his seminal paper [11] on $\mathrm{C}^{*}$-algebras of real rank zero, gave conditions that ensured the linear span of the projections is dense in a wide variety of $\mathrm{C}^{*}$-algebras.

Received by the editors April 16, 1997 and, in revised form, April 21, 1997.

1991 Mathematics Subject Classification. Primary 46L05.

Key words and phrases. Unitarily-invariant spaces, simple $\mathrm{C}^{*}$-algebras.

The first author's research was supported in part by NSERC (Canada).

(C)1998 American Mathematical Society 
The authors would like to thank Prof. G.K. Pedersen for drawing [5] to their attention, and Prof. K.R. Davidson for some helpful comments.

This research was conducted while the first author spent part of his sabbatical leave at University College Cork. He would like to take this opportunity to thank the Department of Mathematics in Cork for their hospitality during his stay.

\section{Closed Lie ideals in $\mathrm{C}^{*}$-Algebras}

Let $A$ be a unital $\mathrm{C}^{*}$-algebra. We denote by $U(A)$ the group of unitaries of $A$ and by $U^{0}(A)$ the connected component of the unit in $U(A)$. It is well known that $U^{0}(A)$ is the subgroup of $U(A)$ generated by the unitaries of the form $e^{i a}$, where $a \in A_{s a}$, a fact that we shall use in the sequel. We shall denote by $Z(A)$ the centre of $A$.

A linear subspace $L$ of $A$ will be said to be invariant under a subset $S$ of $U(A)$ if $u L u^{*} \subseteq L$ for all $u \in S$.

Two examples of unitarily-invariant linear spaces in $A$ that play a crucial role in our investigations are the linear span of the projections, always denoted by $\operatorname{LP}(A)$ in the sequel, and the linear span of the additive commutators $[a, b]=a b-b a$, always denoted by $[A, A]$.

More generally, if $S$ and $T$ are subsets of $A$, we write $[S, T]$ for the linear span of all commutators $[a, b]$, where $a \in S$ and $b \in T$.

Recall that a linear subspace $L$ of $A$ is a Lie ideal if $[a, x]$ belongs to $L$ whenever $a$ belongs to $A$ and $x$ to $L$. We begin by first recording a classical result of I.N. Herstein [7] that we shall use below. We state this result not in its most general form, but in a form sufficient for our purposes.

2.1 Theorem (Herstein). Let $L$ be a Lie ideal in a $C^{*}$-algebra $A$. Then either $L$ is contained in $Z(A)$ or there is a non-zero associative ideal I in A for which $[A, I]$ is contained in $L$.

2.2 Corollary. Let $A$ be a unital, simple $C^{*}$-algebra and $L$ a Lie ideal in $A$. Then $L=0, L=\mathbf{C} 1$ or $[A, A] \subseteq L$.

Proof. In this case $Z(A)=\mathbf{C} 1$ and the only associative ideals of $A$ are 0 and $A$.

The forward implication in the following theorem is well known [11]. We include its proof for the sake of completeness.

2.3 Theorem. Let $L$ be a closed linear subspace of a unital $C^{*}$-algebra $A$. Then $L$ is invariant under $U^{0}(A)$ if and only if $L$ is a Lie ideal in $A$.

Proof. Suppose first that $L$ is invariant under $U^{0}(A)$. If $a \in A_{s a}$ and $x \in L$, set $f(t)=e^{i t a} x e^{-i t a}$ for $t \in \mathbf{R}$. Then the derivative $f^{\prime}(t)$ belongs to $L$ for all $t$ and $f^{\prime}(0)=[i a, x]$. Hence, $[a, x]$ belongs to $L$ for all $a \in A_{s a}$ and therefore for all $a \in A$. Thus, $L$ is a Lie ideal of $A$.

Suppose conversely that $L$ is a Lie ideal. To show that $L$ is invariant under $U^{0}(A)$, we need only show that $u L u^{*} \subseteq L$, if $u=e^{i a}$, where $a \in A_{s a}$. However, it is well known - and easily verified - that the automorphism of $A$ induced by conjugation by $u$ is the exponential of the inner derivation $d=[i a, \cdot]$. Thus, $u x u^{*}=\sum_{n=0}^{\infty} d^{n}(x) / n$ ! for all $x \in A$. Since $L$ is a Lie ideal, it is invariant under $d$ and therefore under $d^{n}$ for all $n$. It follows that if $x \in L$, then $u x u^{*} \in L$.

We shall need the following result of G.K. Pedersen and J. Cuntz [2] at a number of points and we record it here for ease of reference. 
2.4 Theorem (Cuntz-Pedersen). Let $A$ be a unital $C^{*}$-algebra. Then either $A$ has no tracial states and $[A, A]^{-}=A$ or $[A, A]^{-}$is the intersection of the kernels of the tracial states of $A$. In particular, $A$ has at most one tracial state if and only if $\mathbf{C} 1+[A, A]^{-}=A$.

The following result was shown in [9] in the special case of UHF-algebras.

2.5 Theorem. Let $A$ be a unital, simple $C^{*}$-algebra that has at most one tracial state. Then the only closed Lie ideals of $A$ are $0, \mathbf{C} 1,[A, A]^{-}$and $A$.

Proof. If $L$ is a closed Lie ideal, then either $L \subseteq Z(A)=\mathbf{C} 1$ or $[A, A]^{-} \subseteq L$, by Corollary 2.2. Since $[A, A]^{-}$is at most of codimension 1 in $A$, the containment of $[A, A]^{-}$in $L$ implies that $L=[A, A]^{-}$or $L=A$.

The hypothesis of the preceding theorem is, of course, satisfied for a very large class of examples of $\mathrm{C}^{*}$-algebras: for example, UHF-algebras, Bunce-Deddens algebras, finite factors, the irrational rotation algebras $A_{\theta}$, the Cuntz algebras $O_{n}$, and the reduced group $\mathrm{C}^{*}$-algebras $C_{r}\left(\mathbf{F}_{n}\right)$, where $n$ is an integer greater than 1 and $\mathbf{F}_{n}$ is the free group on $n$ generators.

We turn in the next section to the investigation of linear spaces that are not necessarily closed but are invariant under sets of unitaries. These spaces need not be Lie ideals, but we can prove some results for them analogous to those obtained in this section for Lie ideals. However, the method of analysis is quite different from that used in this section.

\section{INVARIANT LINEAR SPACES IN $\mathrm{C}^{*}$-ALGEBRAS}

The following characterisation will be very useful for the considerations that follow.

3.1 Theorem. Let $L$ be a linear subspace of a unital $C^{*}$-algebra $A$ and let $P$ denote the set of projections of $A$. Then the following conditions are equivalent:

(1) $L$ is invariant under the finite-order unitaries of $A$;

(2) $[p, x] \in L$ for all $p \in P$ and $x \in L$;

(3) $p x(1-p) \in L$ for all $p \in P$ and $x \in L$;

(4) $p x q \in L$ for all $p, q \in P$ for which $p q=0$ and for all $x \in L$.

Proof. Suppose that Condition (1) holds. If $p \in P$, then $u=p+i(1-p)$ is a unitary for which $u^{4}=1$. Moreover, $[p, x]=\left(u^{*} x u-u x u^{*}\right) / 2 i$. Hence, if $x \in L$, so is $[p, x]$. Therefore, (1) implies (2).

That (2) implies (3) follows from the equation $p x(1-p)=([p, x]+[p,[p, x]]) / 2$, which holds whenever $p$ is a projection. Also, that (3) implies (4) follows from the equation $p x q=(1-q) p x(1-p) q$, which holds whenever $p$ and $q$ are projections for which $p q=0$.

Finally, suppose that Condition (4) holds and we shall show that (1) follows. For, if $u$ is a finite-order unitary, say $u^{n}=1$, then, by the spectral theorem, we may write $u=\sum_{i=1}^{n} \lambda_{i} p_{i}$, where $\lambda_{1}, \ldots, \lambda_{n}$ are unit-modulus complex numbers and $p_{1}, \ldots, p_{n}$ are pairwise orthogonal projections whose sum is 1 . If $x \in L$, then $u x u^{*}=y+z$, where $y=\sum_{i=1}^{n} p_{i} x p_{i}$ and $z=\sum_{i j} \lambda_{i} \bar{\lambda}_{j} p_{i} x p_{j}$-in this second sum $i, j$ run over all distinct pairs of integers between 1 and $n$. Since, by Condition (4), $p_{i} x p_{j}$ belongs to $L$ if $i \neq j$, we have $z \in L$. Also, $y \in L$ and therefore $u x u^{*} \in L$, since $y=x-\sum_{i j} p_{i} x p_{j}$, where, again, the variables $i, j$ run over all distinct integers between 1 and $n$. Hence, $L$ is invariant under all finite-order unitaries. 
It will follow directly from Theorem 4.2 that each of the above four conditions is equivalent to:

(5) $[e, x] \in L$ for all idempotents $e \in A$ and for all elements $x \in L$.

A linear space invariant under the finite-order unitaries need not be a Lie ideal, nor be invariant under all unitaries. For example, take $A$ to be a non-trivial, unital, simple $\mathrm{C}^{*}$-algebra that has no non-scalar projections (for example, $A=C_{r}\left(\mathbf{F}_{2}\right)$ ). Clearly, in light of Corollary 2.2, we may choose a linear subspace $L$, that we may even take to be closed, that is not a Lie ideal and therefore not unitarily invariant, but which is (trivially) invariant under the finite-order unitaries, since $A$ has no non-scalar finite-order unitaries. (To be specific, if $u$ is a non-scalar unitary in $A$, we may take $L=\mathbf{C} u$.)

Let $M_{n}$ denote the algebra of square matrices over $\mathbf{C}$ of size $n$. Denote by $\left(e_{i j}\right)$ the canonical set of matrix units for $M_{n}$. Thus, if $B$ is a unital $\mathrm{C}^{*}$-algebra, we may identify $M_{n}(B)$ with the tensor product $M_{n} \otimes B$ by identifying the matrix $\left(a_{i j}\right)$ with $\sum_{i, j=1}^{n} e_{i j} \otimes a_{i j}$ in the usual way.

We introduce some notation in this context that we shall use at a number of points in the sequel. Let $c$ be a positive element of $B$ of norm not exceeding 1 and let $i$ and $j$ be distinct indices. Set $c_{i}=c^{1 / 2}$ and $c_{j}=(1-c)^{1 / 2}$. Define an element of $M_{n}(B)$ by the equation

$$
p_{i j}(c)=e_{i i} \otimes c_{i} c_{i}+e_{i j} \otimes c_{i} c_{j}+e_{j i} \otimes c_{j} c_{i}+e_{j j} \otimes c_{j} c_{j} .
$$

It is a simple computation to verify that $p_{i j}(c)$ is a projection.

We shall need the following technical lemma.

3.2 Lemma. Let $B$ be a unital $C^{*}$-algebra and let $A=M_{n}(B)$, where $n>1$. Let $L$ be a linear subpace of $A$ invariant under finite-order unitaries. Suppose also that for all $b \in B$ and for all distinct indices $i, j$, the element $e_{i j} \otimes b$ belongs to $L$. Then $[A, A]$ is contained in $L$.

Proof. If $a, a^{\prime} \in A$, let us write $a \equiv a^{\prime}$ to mean that $a-a^{\prime} \in L$. If $a=$ $\sum_{i, j=1}^{n} e_{i j} \otimes a_{i j}$, then the hypotheses imply that $a \equiv \sum_{i=1}^{n} e_{i i} \otimes a_{i i}$. It follows that to show that all commutators $\left[a, a^{\prime}\right]$ of $A$ belong to $L$, we need only show that elements of the form $e_{i i} \otimes b c-e_{j j} \otimes c b$ belong to $L$ for arbitrary $b, c \in B$. Observe that if $i \neq j$, then $\left[p_{i j}(1 / 2), e_{j i} \otimes b\right]=\left(e_{i i} \otimes b-e_{j j} \otimes b\right) / 2$. Hence, by Theorem 3.1, this commutator belongs to $L$ since $e_{j i} \otimes b$ belongs to $L$. Consequently, for a positive element $c$ of $B$ of norm not exceeding 1 , the commutator $\left[p_{i j}(c), e_{i i} \otimes b-e_{j j} \otimes b\right]$ belongs to $L$. Observe that $\left[p_{i j}(c), e_{i i} \otimes b-e_{j j} \otimes b\right] \equiv e_{i i} \otimes[c, b]-e_{j j} \otimes[b, c]$. It follows from these observations that $e_{i i} \otimes[c, b]$ belongs to $L$ for all postive $c$ of norm not exceeding 1 , and therefore $e_{i i} \otimes[c, b]$ belongs to $L$ for all $c, b \in B$ and all indices $i$. Finally, if $i \neq j$, then $e_{i i} \otimes b c-e_{j j} \otimes c b=e_{i i} \otimes[b, c]+\left(e_{i i} \otimes c b-e_{j j} \otimes c b\right)$, a sum of two elements belonging to $L$, so $e_{i i} \otimes b c-e_{j j} \otimes c b$ belongs to $L$.

3.3 Theorem. Let $B$ be a simple, unital $C^{*}$-algebra and $A=M_{n}(B)$, where $n>1$. Let $L$ be a linear subspace of $A$ invariant under the finite-order unitaries of $A$. Then either $L \subseteq Z(A)$ or $[A, A] \subseteq L$.

Proof. Let $i$ and $j$ be distinct indices and let $I_{i j}$ denote the set of all $b \in B$ for which $e_{i j} \otimes b$ belongs to $L$. Obviously, $I_{i j}$ is a linear subspace of $B$. Moreover, if $c$ is a positive element of $B$ of norm not exceeding 1 , and $b \in I_{i j}$, then $\left[p_{i j}(c), e_{i j} \otimes b\right]$ belongs to $L$. Since $\left(e_{i i} \otimes 1\right)\left[p_{i j}(c), e_{i j} \otimes b\right]\left(e_{j j} \otimes 1\right)=e_{i j} \otimes(c b+b c-b)$, we have $c b+b c$ belongs to $I_{i j}$, by Theorem 3.1. Hence, $b c+c b \in I_{i j}$ for all $b \in I_{i j}$ and 
all $c \in B$. Therefore, $I_{i j}$ is a Jordan ideal of $B$. By simplicity of $B$, there are no non-trivial Jordan ideals [7, Theorem 1], so either $I_{i j}=0$ or $I_{i j}=B$.

Suppose first that $I_{i j}=0$ for all pairs of distinct indices $i, j$. Then, if $a=\left(a_{i j}\right)$ belongs to $L$, we have $a_{i j}=0$ for all $i \neq j$. Let $p=\left(p_{i j}\right)$ be the projection in $A$ defined by setting $p_{i j}=1 / n$ for all $i$ and $j$. Then $[p, a]$ is the matrix with $i j$-th entry equal to $\left(a_{j j}-a_{i i}\right) / n$. Since $[p, a]$ belongs to $L$, by Theorem 3.1, its off-diagonal entries are equal to zero and hence $a_{i i}=a_{j j}$ for all $i$ and $j$. Thus, $a$ has constant diagonal entry and we write this as $b$, so $a_{i i}=b$ for all $i$. Now suppose that $i \neq j$ and that $u$ is an arbitrary unitary element of $B$. The element of $A$ defined by the equation

$$
v=e_{i i} \otimes u+e_{j j} \otimes u^{*}+\sum_{k} e_{k k} \otimes 1
$$

is a unitary - the sum is taken over all indices $k$ between 1 and $n$ that are distinct from $i$ and $j$ and it is understood that the sum is equal to zero if $n=2$. Observe that $v$ is a product of two finite-order unitaries of $A$, since $v=w\left(x w x^{*}\right)$, where $w$ and $x$ are the unitaries defined by the equations

$w=e_{j i} \otimes 1-e_{i j} \otimes 1+\sum_{k} e_{k k} \otimes 1 \quad$ and $\quad x=e_{i j} \otimes 1+e_{j i} \otimes u+\sum_{k} e_{k k} \otimes 1$

and $w^{4}=1$. Again, the sum in these expressions is taken over all indices $k$ between 1 and $n$ that are distinct from $i$ and $j$ and is understood to be equal to zero if $n=2$. Since $L$ is invariant under conjugation by finite-order unitaries, we have $v a v^{*}$ belongs to $L$. It follows that the $i i$-th and $j j$-th entries of $v a v^{*}$ are equal; that is, that $u b u^{*}=u^{*} b u$ and therefore $b$ commutes with $u^{2}$ and $u^{* 2}$. Now choose $u=c+i\left(1-c^{2}\right)^{1 / 2}$, where $c$ is an arbitrary positive element of $B$ of norm not exceeding 1. Then $u^{2}=2 c^{2}-1+2 i c\left(1-c^{2}\right)^{1 / 2}$. Since $b$ commutes with the real part $2 c^{2}-1$ of $u^{2}$, it commutes with $c$. It follows from this that $b \in Z(B)$ and therefore $a \in Z(A)$. Hence, in this case, $L \subseteq Z(A)$.

Now consider the case where at least one of the Jordan ideals $I_{i j}$ is not equal to zero. Then for some pair $k, l$ of distinct indices, $I_{k l}=B$ and therefore $e_{k l} \otimes b$ belongs to $L$ for all $b$ in $B$. Now, for any other pair of distinct indices $i, j$, we may choose a permutation matrix $u$ such that $e_{i j} \otimes b=u\left(e_{k l} \otimes b\right) u^{*}$. Since $u$ is a finite-order unitary, $e_{i j} \otimes b$ belongs to $L$. It follows now from Lemma 3.2 that $[A, A] \subseteq L$.

The preceding theorem does not hold if the restriction to matrix algebras is dropped. For example, the conclusion of the theorem does not hold for $A=C_{r}^{*}\left(\mathbf{F}_{2}\right)$.

\section{The linear span of the projections in a $\mathrm{C}^{*}$-ALgebra}

We begin this section with an application of Lemma 3.2 to obtain a result concerning the linear span of the projections in a matrix algebra.

4.1 Theorem. Let $B$ be a unital $C^{*}$-algebra and $A=M_{n}(B)$, where $n>1$. Then the linear span $L P(A)$ of the projections in $A$ contains $[A, A]$.

Proof. Since $L=L P(A)$ is unitarily invariant, to show $[A, A] \subseteq L$ it suffices to show that $e_{i j} \otimes b \in L$ for all $b \in B$ and all distinct indices $i$ and $j$, by Lemma 3.2. We may suppose $b$ is positive and has norm not exceeding $1 / 2$. By the functional 
calculus, we may write $b=c^{1 / 2}(1-c)^{1 / 2}$, where $c \in B$ and $0 \leq c \leq 1$. Hence, $e_{i j} \otimes b=\left(e_{i i} \otimes 1\right) p_{i j}(c)\left(e_{j j} \otimes 1\right)$ belongs to $L$, by Theorem 3.1.

Let $A$ be a unital $\mathrm{C}^{*}$-algebra and denote by $L I(A)$ the linear span of the idempotents in $A$. The following elementary argument of Herstein's [8, Lemma 1.10] implies that $L I(A)$ is a Lie ideal of $A$. Indeed, if $e=e^{2} \in A$, and if $x \in A$ is arbitrary, then $f_{1}=e+x e-e x e$ and $f_{2}=e+e x-e x e$ are idempotents. Hence $[x, e]=f_{1}-f_{2} \in L I(A)$. That $L I(A)$ is a Lie ideal now follows by linearity.

The following theorem shows that $L I(A)$ coincides with $L P(A)$.

4.2 Theorem. Let $A$ be a unital $C^{*}$-algebra. Then $L P(A)$ contains every idempotent in $A$, and therefore $\operatorname{LP}(A)$ is a Lie ideal.

Proof. Let $L=L P(A)$. If $e$ is an idempotent of $A$, let $p$ be its range projection. Then $p \in A$ and $e p=p$ and $p e=e$. Hence, $e=p+p(e-p)(1-p)$. Thus, to see that $e \in L$ it suffices to show that $p A(1-p) \subseteq L$. We shall show this now and, obviously, to do this we may assume that $p$ is not a scalar. By Theorem 3.1, it suffices to show that $p L(1-p)=p A(1-p)$.

Set $M=p L(1-p)$. Let $u$ be a unitary in the unital $\mathrm{C}^{*}$-algebra $p A p$ and set $v=u+1-p$. Then $v$ is a unitary in $A$ and if $x \in M$, then, since $L$ is unitarily invariant, $v x v^{*}=u x$ belongs to $M$. Using the fact that the unitaries of a $\mathrm{C}^{*}$-algebra linearly span it, we conclude that $a M \subseteq M$ for all $a \in p A p$.

Let $b=p b(1-p) \in A$ with $\|b\|<1$. Then

$$
p_{b}=\left(p-b b^{*}\right)+\left(p-b b^{*}\right)^{\frac{1}{2}} b+b^{*}\left(p-b b^{*}\right)^{\frac{1}{2}}+b^{*} b
$$

is a projection. Thus, by Theorem $3.1, p p_{b}(1-p)=\left(p-b b^{*}\right)^{\frac{1}{2}} b \in M$. Now $\left(p-b b^{*}\right)^{\frac{1}{2}}$ is invertible in $p A p$, say with inverse $d$. Since $p A p M \subseteq M, d p p_{b}(1-p)=b \in M$, and so $p A(1-p)=M$, as required.

The preceding theorem was originally proven by the authors for the case of unital, simple $\mathrm{C}^{*}$-algebras. We would like to thank K.R. Davidson for pointing out to us the construction of the projection $p_{b}$ above, which allowed us to extend our arguments to the general case. We mention also that a somewhat more detailed argument again due to Davidson allows one to show that any idempotent in a unital $\mathrm{C}^{*}$-algebra can actually be written as a linear combination of at most five projections.

The following result is an immediate consequence of Corollary 2.2 and Theorem 4.2 .

4.3 Theorem. Let $A$ be a unital, simple $C^{*}$-algebra. Then $L P(A)=\mathbf{C} 1$ or $[A, A] \subseteq L P(A)$.

If we do not assume that $A$ is a matrix algebra, then $L P(A)$ may not contain $[A, A]$. For instance, let $A$ be the unitisation of the $\mathrm{C}^{*}$-algebra $K$ of all compact operators on a separable, infinite-dimensional Hilbert space. Then $L P(A)$ is easily seen to be the sum of $\mathbf{C} 1$ and the set of finite-rank operators. Since, in this case, $[A, A]=K[5$, Theorem 1.1], it is clear that $L P(A)$ cannot contain $[A, A]$. However, by Theorem 4.1, the linear span of the projections in $M_{2}(A)$ contains all the commutators. From this one easily sees in this case that the linear span of the projections is actually equal to $M_{2}(A)$. 
We give some applications of Theorem 4.3.

4.4 Theorem. Let $A$ be a simple, unital $C^{*}$-algebra with a finite number of extremal tracial states. Suppose furthermore that $[A, A]$ is closed. Then $L P(A)$ is dense in $A$ if and only if $L P(A)=A$.

Proof. One direction is clear. Suppose therefore that $L P(A)$ is dense in $A$. By Theorem 2.4, and the fact that every tracial state is a convex combination of the finitely many extremal tracial states of $A$, the space $[A, A]^{-}$is the intersection of the kernels of these extremal states. Hence, $[A, A]=[A, A]^{-}$is finite-codimensional in $A$. Then $L P(A)$ is the sum of $[A, A]$ and a finite dimensional space, since by Theorem 4.3, $[A, A]$ is contained in $\operatorname{LP}(A)$. It follows that $L P(A)$ is closed in $A$. Consequently, $\operatorname{LP}(A)=A$.

The following result of $\mathrm{K}$. Thomsen [12] provides a large class of simple, unital $\mathrm{C}^{*}$-algebras $A$ for which the commutator space $[A, A]$ is closed. We refer the reader to [4] for the definition of the covering dimension of a compact Hausdorff space.

4.5 Theorem (Thomsen). Let $A=\lim _{i \rightarrow \infty}\left(A_{i}, \phi_{i}\right)$ be a simple, infinite-dimensional, unital inductive limit of $C^{*}$-algebras, where each of the $A_{i}$ 's is of the form

$$
A_{i}=C\left(X_{i 1}\right) \otimes M_{t(i, 1)} \oplus C\left(X_{i 2}\right) \otimes M_{t(i, 2)} \oplus \ldots \oplus C\left(X_{i n_{i}}\right) \otimes M_{t\left(i, n_{i}\right)},
$$

and each $X_{i k}$ is a compact, connected Hausdorff space. Assume there is some nonnegative integer $d$ such that the covering dimension $\operatorname{dim}\left(X_{i k}\right) \leq d$ for all $i$ and $k$. If $a \in A_{\text {sa }}$ and $\tau(a)=0$ for all tracial states $\tau$ on $A$, then there are $d+7$ elements $x_{i}$ in $A$ such that $a=\sum_{i=1}^{d+7}\left[x_{i}, x_{i}^{*}\right]$.

It follows by combining Theorems 2.4 and 4.5 that if $A$ is a simple, unital inductive limit of $\mathrm{C}^{*}$-algebras of the above type, then $[A, A]$ is closed. As is well known, many $\mathrm{C}^{*}$-algebras originally defined by other means have been shown to be inductive limits of this type, in fact with each $X_{i k}$ equal to the set of complex numbers of modulus one. (Note that $d=1$ in this case.) Examples include the Bunce-Deddens algebras [6] and the irrational rotation algebras [3].

Thomsen's theorem generalises an earlier result of T. Fack [5, Theorem 3.1], who considered the case where $A$ is a simple, unital AF $\mathrm{C}^{*}$-algebra; in this case each $X_{i k}$ consists of a single point.

4.6 Theorem. The following $C^{*}$-algebras are linearly spanned by their projections:

(1) the Bunce-Deddens algebras;

(2) the irrational rotation algebras;

(3) simple, unital $A F C^{*}$-algebras with finitely many extremal tracial states;

(4) UHF $C^{*}$-algebras.

Proof. (1) and (2): In view of the preceding comments, we see that $[A, A]$ is closed in both cases. Furthermore, in both cases, the algebras are simple, unital and possess a unique tracial state. Since they also possess non-trivial projections, it follows from [11, Corollary 4] that the linear span of the projections is dense, and by Theorem $4.3, L P(A)$ contains $[A, A]$. Hence, $L P(A)=A$ by Theorem 4.4 .

(3): That the projections span a dense linear subspace is clear. That $[A, A]$ is closed follows from the above-mentioned result of Fack [5, Theorem 3.1]. Again, Theorem 4.3 implies that $L P(A)$ contains $[A, A]$, and the result follows from Theorem 4.4 .

(4): This is a special case of (3), as is well known [10, p. 180]. 
4.7 Theorem. Let $A$ be an infinite, simple, unital $C^{*}$-algebra. Then $L P(A)=A$.

Proof. It is a consequence of [5, Corollary 2.2] that $A=[A, A]$. Moreover, since $A$ is infinite, the unit 1 is equivalent to a proper projection, which cannot therefore be a scalar. Hence, $L P(A) \nsubseteq \mathbf{C}$ 1. It follows now from Theorem 4.3 that $L P(A)=A$.

In particular, Theorem 4.7 implies that the Cuntz algebras $O_{n}$ are linearly spanned by their projections.

Observe that if $A$ is a unital $\mathrm{C}^{*}$-algebra and $L P(A)=A$, then any linear subspace $L$ invariant under finite order unitaries is a Lie ideal of $A$. For, by Theorem 3.1, $[p, x] \in L$ if $x \in L$ for all projections $p$, and so by linearity, $[a, x] \in L$ for all $a \in L P(A)=A$.

If $A$ is a simple, unital $\mathrm{C}^{*}$-algebra, we may have $L P(A) \neq A$, even if $A$ has non-scalar projections. For example, B.E. Blackadar [1] has given an example of a simple, unital $\mathrm{C}^{*}$-algebra $A$ having exactly one equivalence class of non-scalar projections and two extremal tracial states. Hence, if $p$ is a non-scalar projection in $A$, there exists an element $u$ of $A$ for which $p=u^{*} u$ and $1-p=u u^{*}$. Therefore, $1-2 p=\left[u, u^{*}\right]$ and so $p \in \mathbf{C} 1+[A, A]$. Hence, $L P(A) \subseteq \mathbf{C} 1+[A, A]$. The reverse inclusion also holds, by Theorem 4.3. Hence, $L P(A)=\mathbf{C} 1+[A, A]$. Clearly, $[A, A]^{-}$ is contained in the intersection of the kernels of the two extremal tracial states and therefore $[A, A]^{-}$is of at least codimension 2 in $A$. Consequently, $\operatorname{LP}(A) \neq A$.

We finish with an observation related to the considerations of this paper. Pedersen showed in [11] that if $A$ is a simple, unital $\mathrm{C}^{*}$-algebra with non-scalar projections, then the subalgebra $B$ of $A$ generated by the projections is dense in $A$. We shall show that $B=A$. In fact, we show a more general result: suppose $A$ is a unital $\mathrm{C}^{*}$-algebra which contains a projection $p$ such that the ideals generated by $p$ and $1-p$, respectively, are equal to $A$. (This is obviously true where $A$ is simple, if $A$ contains a non-scalar projection.) Since $p A(1-p) \subseteq L P(A)$, as we saw in the proof of Theorem 4.2, we have $p A(1-p) A p \subseteq B$. But $A(1-p) A=A$, and so $p A p \subseteq B$. Similarly, $(1-p) A(1-p) \subseteq B$. Also, $(1-p) A p \subseteq L P(A) \subseteq B$. Combining these inclusions, we have $A \subseteq B$ and therefore $A=B$.

This fails even for primitive $\mathrm{C}^{*}$-algebras. Indeed, if we take $A$ to be the unitisation of the compact operators acting on an infinite dimensional separable Hilbert space, then the algebra generated by the projections is just the set of operators of the form finite rank plus scalar. Clearly it is dense, but not the entire algebra.

\section{REFERENCES}

1. B.E. Blackadar, A simple unital projectionless $C^{*}$-algebra, J. Operator Theory 5 (1981), 6371. MR 82h: 46076

2. J. Cuntz and G.K. Pedersen, Equivalence and traces on $C^{*}$-algebras, J. Funct. Anal. 33 (1979), 135-164. MR 80m:46053

3. G.A. Elliott and D. Evans, The structure of the irrational rotation $C^{*}$-algebra, Ann. Math. 138 (1993), 477-501. MR 94j:46066

4. R. Engelking, Dimension Theory, North-Holland, Amsterdam, Oxford and New York, 1978. MR 58:2753b

5. T. Fack, Finite sums of commutators in $C^{*}$-algebras, Ann. Inst. Fourier, Grenoble 32 (1982), 129-137. MR 83g:46051

6. P.G. Ghatage and W.J. Phillips, $C^{*}$-algebras generated by weighted shifts II, Indiana Univ. Math. J. 30 (1981), 539-545. MR 83h:46072

7. I.N. Herstein, On the Lie and Jordan rings of a simple associative ring, Amer. J. Math. $\mathbf{7 7}$ (1955), 279-285. 
8. I.N. Herstein, Topics in Ring Theory, Chicago Lecture Notes in Mathematics, University of Chicago Press, Chicago and London, 1969. MR 42:6018

9. L. Marcoux, On the closed Lie ideals of certain $C^{*}$-algebras, J. Integr. Equat. Oper. Th. 22 (1995), 463-475. MR 96f:46123

10. G.J. Murphy, $C^{*}$-Algebras and Operator Theory, Academic Press, San Diego, 1990. MR 91 m:46084

11. G.K. Pedersen, The linear span of projections in simple $C^{*}$-algebras, J. of Operator Theory 9 (1980), 289-296. MR 82b:46078

12. K. Thomsen, Finite sums and products of commutators in inductive limit $C^{*}$-algebras, Ann. Inst. Fourier, Grenoble 43 (1993), 225-249. MR 94g:46062

Department of Mathematical Sciences, University of Alberta, Edmonton, Alberta, CANADA T6G 2G1

E-mail address: 1.marcoux@ualberta.ca

Department of Mathematics, University College, Cork, Ireland

E-mail address: gjm@ucc.ie 